\title{
Current treatment options for acanthosis nigricans
}

This article was published in the following Dove Press journal:

Clinical, Cosmetic and Investigational Dermatology

\author{
Nupur U Patel' \\ Catherine Roach' \\ Hossein Alinia' \\ William W Huang' \\ Steven R Feldman ${ }^{1-3}$ \\ 'Department of Dermatology, Center \\ for Dermatology Research, Wake \\ Forest University School of Medicine, \\ Winston-Salem, NC, ${ }^{2}$ Department of \\ Pathology, Wake Forest University \\ School of Medicine, Winston-Salem, \\ NC, ${ }^{3}$ Department of Public Health \\ Sciences, Wake Forest University \\ School of Medicine, Winston-Salem, \\ NC, USA
}

\begin{abstract}
Acanthosis nigricans (AN) is a common dermatologic manifestation of systemic disease that is associated with insulin resistance, diabetes mellitus, obesity, internal malignancy, endocrine disorders, and drug reactions. Treatment of AN primarily focuses on resolution of the underlying disease processes causing the velvety, hyperpigmented, hyperkeratotic plaques found on the skin. While the goal of therapy is to treat the primary cause, cosmetic resolution of AN lesions can be important for patients and their quality of life. Treatment options for AN have not been extensively studied; however, smaller powered clinical trials and case reports exist in the literature. Our review aims to explore and evaluate the current treatment options that exist for AN.

Keywords: acanthosis nigricans, diabetes, obesity, hyperinsulinemia, dermatologic condition, dermatology, malignant acanthosis, pseudoacanthosis, metformin, isotretinoin, tretinoin, topical therapy
\end{abstract}

\section{Background}

Acanthosis nigricans (AN) is a common cutaneous condition that can be a manifestation of systemic disease that is associated with insulin resistance, diabetes mellitus, obesity, internal malignancy, endocrine disorders, and drug reactions. It is characterized by thick velvety hyperpigmented plaques of the intertriginous, flexural, and less commonly extensor areas of the skin. ${ }^{1}$ When AN is associated with internal malignancy, skin changes develop rapidly and are often accompanied by mucosal involvement, seborrheic keratoses, acrochordons, and tripe palms. In contrast, endocrinopathy-associated AN develops more insidiously and covers a smaller area of the body. Patients are often obese and may have a history or family history of diabetes or polycystic ovarian syndrome. Prevalence of AN among primary care patients in the USA has been reported to be as high as $19.4 \% .^{2}$ High-risk populations are African-Americans, Hispanics, and Native Americans, although this condition presents in all races.

The severity of AN has a positive correlation with fasting insulin levels. ${ }^{3} \mathrm{AN}$ is likely the result of high levels of insulin binding to insulin-like growth factor 1 receptors (IGF-1Rs) on keratinocytes and fibroblasts. This leads to a proliferation of both cell types and clinically apparent plaques and papillomatosis. ${ }^{3}$ In addition, high levels of insulin increase the amount of circulating free IGF-1, which results in keratinocyte growth and differentiation.

Treatment of AN should focus on correction of the underlying disease process. ${ }^{4}$ Weight reduction in obesity-related AN may resolve hyperkeratotic lesions, while
Correspondence: Hossein Alinia Department of Dermatology, Center for Dermatology Research, Wake Forest University School of Medicine, 4618 Country Club Road, Winston-Salem, NC 27I04, USA

$\mathrm{Tel}+\mid 3367161763$

Fax +I 3367167732

Email haliniamd@gmail.com 
correction of hyperinsulinemia can reduce the burden of AN lesions. ${ }^{4}$ Cessation of the causative agent in drug-induced $\mathrm{AN}$ often results in resolution of $\mathrm{AN}$, and surgical removal of tumors is the mainstay of treatment in malignancy-associated AN. ${ }^{5}$ While the goal of therapy for AN is to treat the primary cause, cosmetic resolution of AN lesions can be important for patients and their quality of life. Treatment options have not been extensively studied; however, smaller powered clinical trials and case reports exist in the literature and can aid providers in creating treatment plans for patients with AN. This article aims to explore and evaluate the current treatment options that exist for AN.

A literature search through PubMed was performed using MeSH key words "acanthosis nigricans", "treatment options", "metformin", "isotretinoin", "acitretin", "topical therapy", and "laser". After reviewing search results for inclusion, a total of 19 articles were retrieved using these keywords in combinations on PubMed. Further articles were found using source materials from the references reviewed and chosen for inclusion. Articles and case reports that focused specifically on AN and its treatment were given preference. Studies and articles published from 1951 to 2017 were used in order to encompass the breadth of the research that has been conducted on the subject. Clinical trial evidence for five recent trials was found using the phrases "acanthosis nigricans" and "treatment" on Clinicaltrials.gov. Review articles and the authors' experiences were drawn upon to create the discussion section.

\section{Topical treatments Topical retinoids}

Topical retinoids are considered one of the first-line treatment options for AN. ${ }^{3}$ In a study of 30 patients, clinical improvement of treatment-resistant $\mathrm{AN}$ was seen in all patients after 14 days of $0.05 \%$ tretinoin application ${ }^{6}$ (Table 1 ). Of the 30 patients, 24 $(80 \%)$ showed total clearance at 16 weeks. However, intermittent tretinoin was needed to maintain improvement as relapse was noted within a period of 4 weeks after discontinuation of treatment. Histopathologically, the typical changes of hyperkeratosis and keratotic material between papillae were resolved on biopsy after 8 weeks of tretinoin application.

Use of topical $0.1 \%$ tretinoin also improved $\mathrm{AN}$ as shown in two case reports. An 18-year-old woman with AN experienced clearance of her neck AN in 10 days, followed by improvement in color and hyperkeratosis of her axillae within 2 weeks. ${ }^{7}$ Another patient experienced clearing of left axilla $\mathrm{AN}$ after tretinoin $0.1 \%$ gel was applied twice daily for 2 weeks, while the right axilla was used as a control and did not show any improvement. ${ }^{8}$

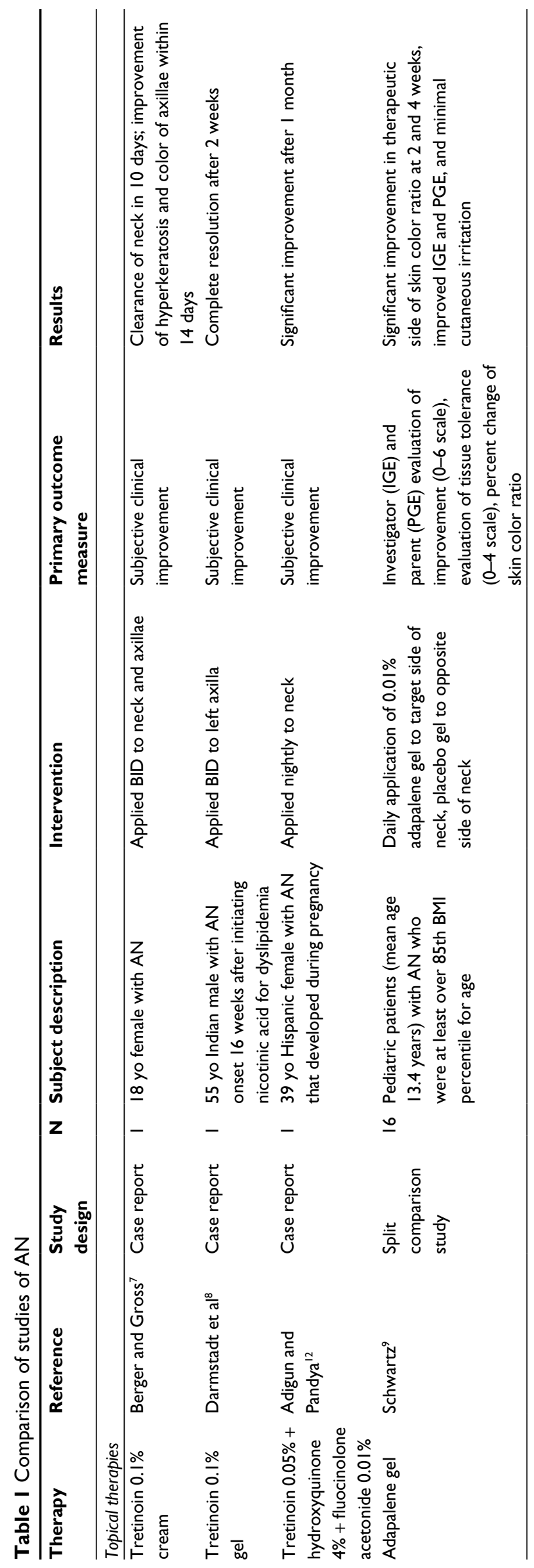




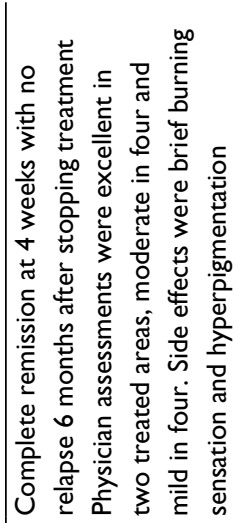

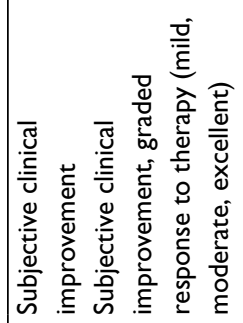

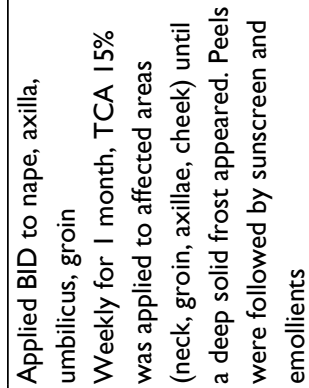

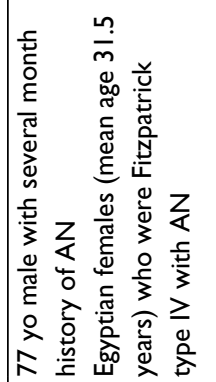

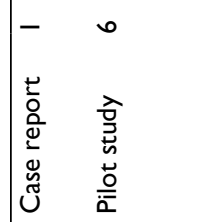

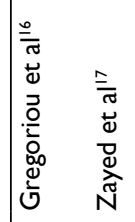

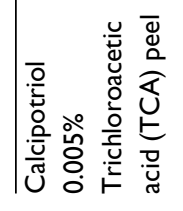

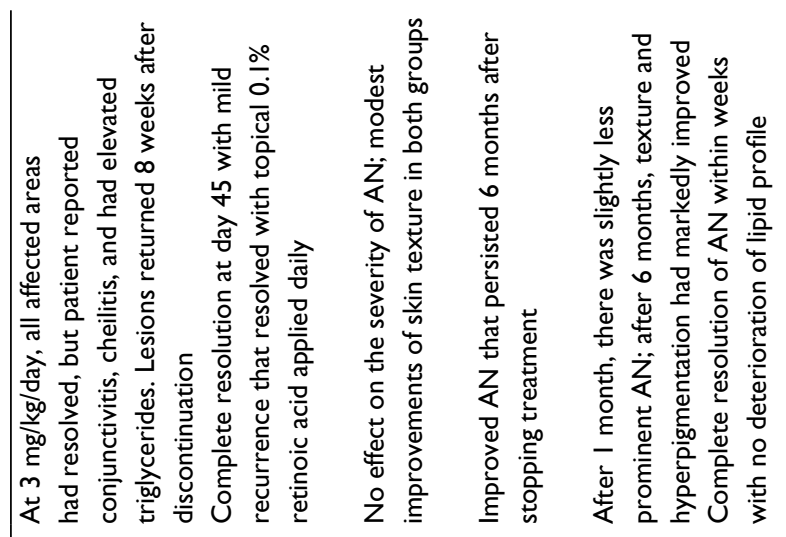

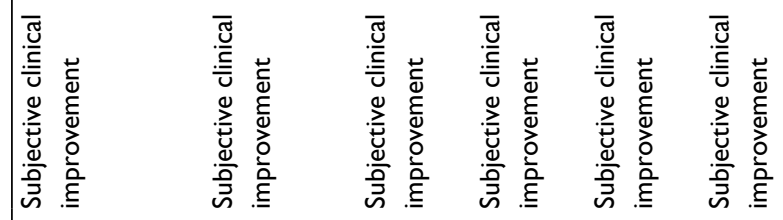

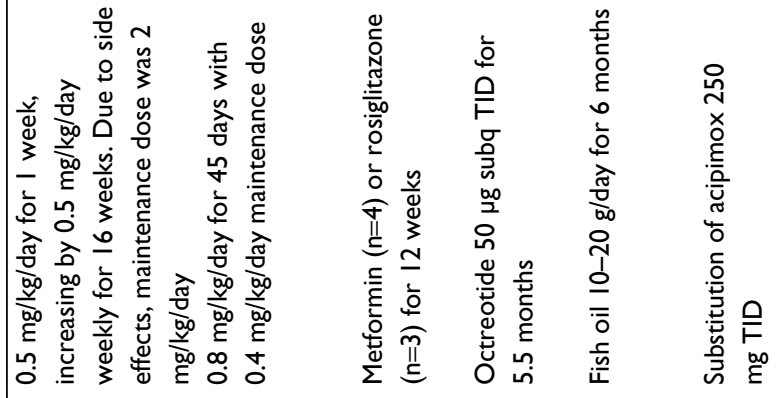

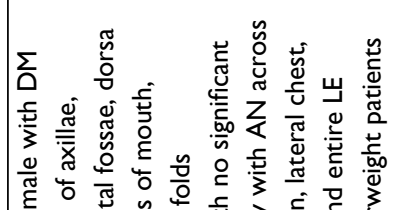

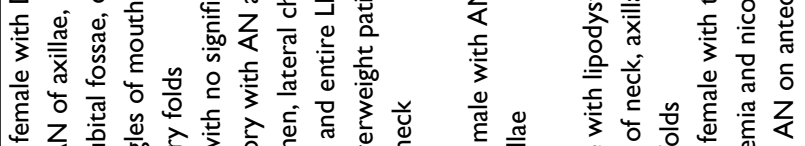

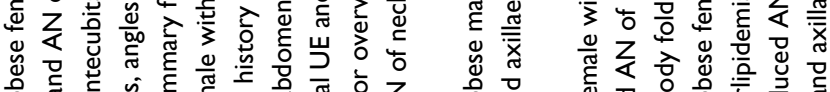

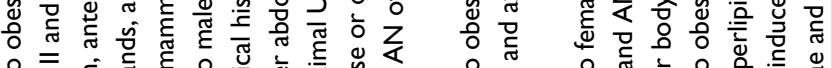

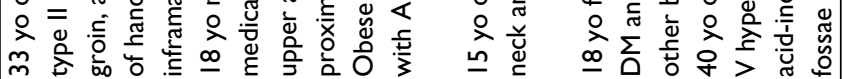

-

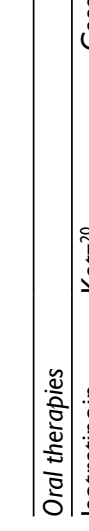

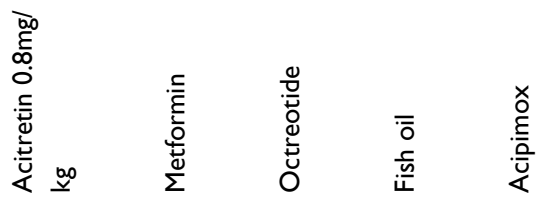

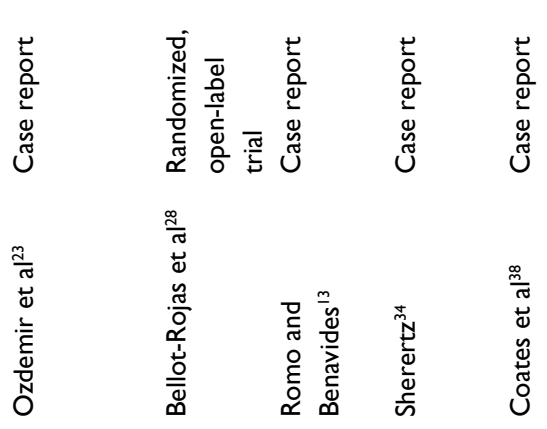

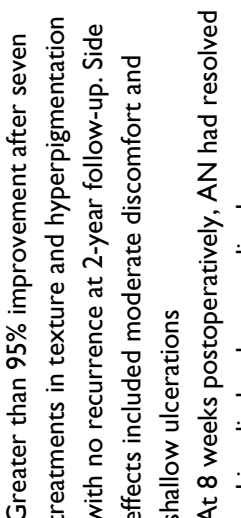

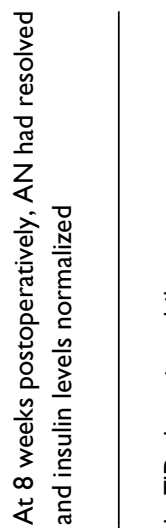

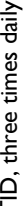


Two studies that used adapalene gel for childhood AN showed variable results. ${ }^{9,10}$ One randomized controlled trial had 16 children in Thailand use $0.1 \%$ adapalene to one side of their neck affected by AN with a minimization of skin darkening found to result. The therapeutic effect was attributed to an alteration in epidermal keratinization, and little to no skin irritation occurred. ${ }^{9}$ Another split site comparison study of 16 pediatric AN patients treated with $0.1 \%$ adapalene gel in India showed $60.7 \% \pm 28.5 \%$ improvement in the mean skin color ratio, defined as ([Felix von Luschan skin color at neck - interscapular skin color]/interscapular skin color). The therapeutic side showed significant improvement as measured by a significant decrease in the mean skin color ratio compared to the untreated side at weeks 2 and 4, respectively. ${ }^{10}$

Combination therapy may also be used to successfully treat AN. In one case report, the combination of $0.05 \%$ tretinoin cream and $12 \%$ ammonium lactate cream led to resolution of obesity-related AN. ${ }^{11}$ In another case report of idiopathic AN, a triple-combination depigmenting cream composed of $0.05 \%$ tretinoin, $4 \%$ hydroquinone, and $0.01 \%$ fluocinolone acetonide was applied at night along with daily sunscreen, which showed successful results after 1 month of use. ${ }^{12}$

\section{Topical vitamin D analogs}

Calcipotriene, a vitamin D analog, is another topical treatment option for AN. It is thought to inhibit keratinocyte proliferation and promote differentiation by increasing keratinocyte intracellular calcium and cyclic GMP levels. ${ }^{3}$ By reducing the number of keratinocytes, it may minimize the cutaneous effects of insulin. ${ }^{13}$ One report in the literature demonstrated improvement of a mixed-type $\mathrm{AN}$ in the flexural areas of an obese man after 3 months of $0.005 \%$ calcipotriol cream application twice daily. ${ }^{3,14}$ Another report showed that an obese woman with AN showed improvement in her lesions with calcipotriol ointment use twice daily, which was also for a period of 3 months. ${ }^{15}$ Gregoriou et al reported calcipotriol to be a safe, effective, and well-tolerated treatment for AN, particularly when etiological treatment is not an option. ${ }^{3,16}$

\section{Chemical peels}

Although considered cosmetic in nature, superficial chemical peels are a relatively safe and effective treatment option for AN. Trichloroacetic acid (TCA) is a chemical exfoliating agent that causes destruction of the epidermis with subsequent repair and rejuvenation. ${ }^{3}$ As a caustic substance, TCA causes coagulation and precipitation of skin proteins, leading to necrosis of epidermis. This destruction is followed by inflammation and the activation of wound repair, causing re-epithelialization with smoother skin. ${ }^{3}$ Zayed et al reported improvement in AN in six female patients who used TCA peels in a pilot study. Improvement was seen with regards to hyperpigmentation, thickening, and overall appearance. ${ }^{17}$ TCA has several advantages: it is safe, easily accessible, inexpensive, and can be easily prepared. Furthermore, TCA is also a stable compound with a known precipitation, absorption, and peel depth, which make judging its endpoint exfoliation simple. ${ }^{3}$

\section{Oral treatment}

\section{Oral retinoids (isotretinoin and acitretin)}

Oral retinoids such as isotretinoin and acitretin can also be effective treatment options for AN. However, improvement requires large doses and extended courses, with relapses described in the literature. ${ }^{1,3,18}$ One proposed mechanism of action for these drugs is normalization of epithelial growth and differentiation. ${ }^{3,19}$ Extensive AN associated with obesity has been successfully treated with isotretinoin ( $3 \mathrm{mg} / \mathrm{kg} /$ day), but relapsed when treatment was discontinued. ${ }^{20}$ Another patient noted $90 \%$ improvement of palmar AN and 50\% improvement of axillary AN within 2 months of taking isotretinoin $80 \mathrm{mg} /$ day. After gradually tapering this dose over more than a year and receiving over a total of $30 \mathrm{~g}$, the patient experienced recurrence of his skin lesions that improved on taking 1000 mg metformin twice daily. ${ }^{21}$ Another case report of a 17-yearold female with Costello syndrome showed improvement in $\mathrm{AN}$ of the neck and dorsum of the hands as a result of oral isotretinoin treatment started for nodulocystic acne. ${ }^{22}$

Fewer reports of AN treatment with acitretin are present in the literature; however, those that exist report success in cases of syndromic and benign AN. ${ }^{3}$ One case showed that an 18-year-old male with generalized idiopathic AN experienced complete recovery after 45 days of acitretin $0.8 \mathrm{mg} / \mathrm{kg}(50$ $\mathrm{mg}$ ) divided into two daily doses. After starting maintenance therapy of $25 \mathrm{mg}$ acitretin daily for 2 months, lesions recurred that subsequently resolved with topical application of $0.1 \%$ retinoic acid. ${ }^{23}$ Because of acitretin's longer terminal elimination half-life and fewer lipophilic properties, its use may be limited, causing potential for early recurrence. ${ }^{3,24}$ One case report has also been published on the successful treatment of AN with oral etretinate, a second-generation systemic retinoid in a patient with generalized lipodystrophy.

\section{Metformin and rosiglitazone}

For the treatment of AN associated with insulin resistance, conventional insulin-sensitizing agents such as metformin can be used. Metformin increases peripheral insulin responsiveness, resulting in reduction of glucose production, 
hyperinsulinemia, body weight, and fat mass, as well as an increase in insulin sensitivity in patients with insulin resistance and AN. ${ }^{3,18,25,26}$ One recent clinical trial conducted in India treated 40 patients with $\mathrm{AN}$ and insulin resistance (detected by the Homeostatic Model Assessment for Insulin Resistance) with $500 \mathrm{mg}$ metformin thrice daily for 3 months. Compared with 20 control patients who were given placebo, patients treated with metformin demonstrated statistically and clinically significant improvement in AN of the neck and axilla, but not in AN of the knuckles, fingers, or elbows. In one published case series, three obese adolescent patients were started on metformin and dietary modifications. All the patients had previously failed topical therapy for AN, either with calcipotriol or with corticosteroids. Improvement was subjectively seen in all the three patients with no relapse reported after 1 year. ${ }^{13,18}$ Another case report of a 14-year-old boy treated with $850 \mathrm{mg}$ metformin twice daily reported no resolution of AN after 6 months of treatment. ${ }^{27}$

A prospective, randomized, open-label trial compared metformin and rosiglitazone in 27 insulin-resistant patients for 12 weeks. While a greater reduction in fasting insulin levels was seen with rosiglitazone, only minimal improvement in AN lesions and skin texture was seen with either agents. ${ }^{28}$ Duration of treatment may also play a role in observing clinical changes to the skin, as metformin does improve both AN and insulin resistance when used for 6 or more months. ${ }^{3,25}$ In a smaller prospective 6-month trial of metformin in patients with $\mathrm{AN}$ and insulin resistance, improvement in AN resulted in three of five patients (two adolescents and one adult). ${ }^{25}$

\section{Other oral agents}

A combination of metformin with thiazolidiones, medications that increase insulin sensitivity in peripheral muscles, has also been reported to have good results in patients with AN. ${ }^{3,29,30}$ Hyperandrogenemia, insulin resistance, and AN syndrome (HAIR-AN syndrome) patients may also be treated with a combination of metformin and oral contraceptives. ${ }^{1,31}$ The spontaneous regression of AN has also been reported after the addition of TZD pioglitazone and sitagliptin, a dipeptidyl peptidase 4 inhibitor which increases insulin secretion, in an insulin-resistant patient. ${ }^{32}$ The long-term use of ocreotide, a synthetic analog of somatostatin, sustained improvement of $\mathrm{AN}$ and reduction of body weight in a severely obese boy with insulin resistance 6 months after treatment cessation. ${ }^{33}$

\section{Other therapies}

Other therapies that have improved AN according to published case reports include fish oil, podophyllin, and combination therapy with urea, salicylic acid, and triplecombination depigmenting cream. Fish oil containing omega-3 fatty acids improved hyperpigmentation and skin texture in one woman with $\mathrm{AN}$ and a lipodystrophic form of diabetes after 6 months of treatment in spite of continued therapy with niacin. ${ }^{1,34}$ Podophyllin $20 \%$ in alcohol has been reported to temporarily resolve AN lesions of the hands; however, resolution was preceded by a local reaction after application of the solution. ${ }^{1,35}$ Other treatment options that have been reported with variable results include topical urea and salicylic acid application. ${ }^{3,4}$

Use of alexandrite laser is another cosmetic treatment option that has been demonstrated to be effective in improving AN lesions. Greater than $95 \%$ clearance in the left axilla was observed after seven treatments with long-pulsed (5 msec) alexandrite laser at 4-8 weeks interval..$^{36}$ This laser was designed to target melanin in hair and was hypothesized to improve the darkening of the skin in affected areas. Although it is not cost-effective compared to other topical and oral treatment options, the use of alexandrite laser for treatment of $\mathrm{AN}$ is promising for the future.

\section{Discussion}

Current treatment paradigms for AN are shifting to include addressing the cutaneous pathology as well as the underlying disease or drug. Non-medication options include weight loss and exercise to increase insulin sensitivity in insulinresistant AN. ${ }^{3}$ Treatment of AN is multifactorial because the skin findings are often accompanied by underlying disease. Initial considerations for the AN workup include evaluating patients for insulin resistance syndrome characterized by obesity, dyslipidemia, hypertension, and diabetes mellitus type II. Providers should obtain a fasting lipid panel, glucose, and insulin, as well as a complete blood count and liver function testing. If malignancy-associated AN is considered, imaging studies such as plain radiography and computerized tomography/magnetic resonance imaging may provide valuable information. ${ }^{3}$ Oral metformin and combinations of other insulin-mediating medications are effective, as they are therapies aimed at resolving the underlying causes contributing to AN.

For cosmetic treatment, topical retinoids are considered the first-line therapy for insulin-resistant AN by modifying keratinization rate. However, topical tretinoin requires application for long durations and improves hyperkeratosis, but not hyperpigmentation. ${ }^{3}$ Topical salicylic acid, podophyllin, urea, and calcipotriol also require frequent application, while TCA peels may provide a faster and less time-intense burden. ${ }^{3}$ 
Other options include dermabrasion or alexandrite laser; however, cost must be considered with these treatments as well the potential for post-inflammatory hyperpigmentation.

An important consideration when evaluating a patient with $\mathrm{AN}$ is the potential for psychological distress. In one study, obese female adolescents with AN showed significantly lower self-esteem status than the healthy control. Testosterone levels significantly correlated with poor self-esteem scores among the obese females with AN. ${ }^{37}$ While correction of underlying causes remains the mainstay of treatment, it is important to consider cosmetic options to improve patient quality of life that may be affected by the presence of AN lesions.

Future studies of AN are currently being conducted, with several trials registered on Clinicaltrials.gov. These include a study of metformin on the treatment of AN in children (NCT02438020), an examination of glucose intolerance in lean children with a family history of diabetes and obese children with AN (NCT00000112), a spectroscopic and colorimetric analysis of AN in hyperinsulinemia patients (NCT01125150), a study of leuprolide acetate and spironolactone on insulin resistant women with hyperandrogenism insulin resistance AN syndrome (NCT00004311), and a study of the effect of the dietary supplement melatonin on obese patients with AN (NCT02604095). While these studies have the potential to shed light on therapy for AN, clinical trials are needed to examine topical and oral treatment options specifically for the improvement of AN skin lesions. The current literature that exists is limited, and higher powered studies with larger patient populations are needed to further elucidate the AN treatment paradigm.

\section{Conclusion}

We identified various treatment options for AN including oral, topical and chemical peels. While cosmetic concerns are important aspects of AN treatment, providers must attempt to discover and address any underlying pathology such as diabetes mellitus and cancer. Currently no single treatment of AN is superior; however, as the pathogenesis of AN is further elucidated, more targeted therapies of superficial lesions and associated pathology will greatly benefit these patients.

This review is intended to provide medical professionals with broad treatment options for patients with AN as well as considerations for etiology of the condition. Although the search strategy was designed to capture all peer-reviewed literature of AN treatment, there is the possibility for missing articles. Improved patient understanding of AN and its etiology should be a mainstay of management. Although cosmetic outcomes are an important component of $\mathrm{AN}$, recognition and treatment of the underlying pathology is paramount. Future research in the pathogenesis of insulin-resistant AN and malignancy-related AN will further the development of targeted AN therapies. With more information on therapies that treat the underlying causes of AN, as well as treatment options for the cosmetic appearance of AN lesions, providers will have the opportunity to treat patients underlying diseases and the psychological consequences of the AN to improve patient quality of life.

\section{Disclosure}

Dr Feldman is a speaker for Janssen and Taro. He is a consultant and speaker for Galderma, Stiefel/GlaxoSmithKline, Abbott Labs, and Leo Pharma Inc. Dr Feldman has received grants from Galderma, Janssen, Abbott Labs, Amgen, Stiefel/ GlaxoSmithKline, Celgene, and Anacor. He is a consultant for Amgen, Baxter, Caremark, Gerson Lehrman Group, Guidepoint Global, Hanall Pharmaceutical Co Ltd, Kikaku, Lilly, Merck \& Co Inc, Merz Pharmaceuticals, Mylan, Novartis Pharmaceuticals, Pfizer Inc, Qurient, Suncare Research, and Xenoport. He is on an advisory board for Pfizer Inc. Dr Feldman is the founder and holds stock in Causa Research and is majority owner in Medical Quality Enhancement Corporation. He receives royalties from UpToDate and Xlibris. Dr Patel, Dr Roach, Dr Alinia, and Dr Huang report no conflicts of interest in this work.

\section{References}

1. Higgins SP, Freemark M, Prose NS. Acanthosis nigricans: a practical approach to evaluation and management - eScholarship. Dermatol Online J. 2008;14(9):2.

2. Kong AS, Williams RL, Rhyne R, et al. Acanthosis nigricans: high prevalence and association with diabetes in a practice-based research network consortium - a PRImary care Multi-Ethnic Network (PRIME Net) Study. J Am Board Fam Med. 2010;23(4):476-485.

3. Phiske MM. An approach to acanthosis nigricans. Indian Dermatol Online J. 2014;5(3):239-249.

4. Puri N. A study of pathogenesis of acanthosis nigricans and its clinical implications. Indian J Dermatol. 2011;56(6):678.

5. Ghosh S, Roychowdhury B, Mukhopadhyay S, Chowdhury S. Clearance of acanthosis nigricans associated with insulinoma following surgical resection. QJM. 2008;101(11):899-900.

6. Lahiri K, Malakar S [webpage on the Internet]. Topical tretinoin in acanthosis nigricans. Indian J Dermatol Venereol Leprol. 62(3):159-161. http:// www.ncbi.nlm.nih.gov/pubmed/20948022. Accessed October 27, 2017.

7. Berger BJ, Gross PR. Another use for tretinoin - pseudoacanthosis nigricans. Arch Dermatol. 1973;108(1):133-134.

8. Darmstadt GL, Yokel BK, Horn TD. Treatment of acanthosis nigricans with tretinoin. Arch Dermatol. 1991;127(8):1139-1140.

9. Schwartz RA. Efficacy of topical $0.1 \%$ adapalene gel for use in the treatment of childhood acanthosis nigricans: a pilot study. Dermatol Ther. 2015;28(4):266-266.

10. Treesirichod A, Chaithirayanon S, Wongjitrat N, Wattanapan P. The efficacy of topical $0.1 \%$ adapalene gel for use in the treatment of childhood acanthosis nigricans: a pilot study. Indian J Dermatol. 2015;60(1):103. 
11. Blobstein SH. Topical therapy with tretinoin and ammonium lactate for acanthosis nigricans associated with obesity. Cutis. 2003;71(1): 33-34.

12. Adigun CG, Pandya AG. Improvement of idiopathic acanthosis nigricans with a triple combination depigmenting cream. J Eur Acad Dermatol Venereol. 2009;23(4):486-487.

13. Romo A, Benavides S. Treatment options in insulin resistance obesityrelated acanthosis nigricans. Ann Pharmacother. 2008;42(7-8) 1090-1094.

14. Böhm M, Luger TA, Metze D. Treatment of mixed-type acanthosis nigricans with topical calcipotriol. Br J Dermatol. 1998;139(5):932-934.

15. Lee H-W, Chang S-E, Lee M-W, Choi J-H, Moon K-C, Koh J-K. Hyperkeratosis of the nipple associated with acanthosis nigricans: treatment with topical calcipotriol. J Am Acad Dermatol. 2005;52(3):529-530.

16. Gregoriou S, Anyfandakis V, Kontoleon P, Christofidou E, Rigopoulos D, Kontochristopoulos G. Acanthosis nigricans associated with primary hypogonadism: successful treatment with topical calcipotriol. J Dermatolog Treat. 2008;19(6):373-375.

17. Zayed A, Sobhi RM, Abdel Halim DM. Using trichloroacetic acid in the treatment of acanthosis nigricans: a pilot study. J Dermatolog Treat. 2014;25(3):223-225.

18. Hermanns-Lê T, Scheen A, Piérard GE. Acanthosis nigricans associated with insulin resistance: pathophysiology and management. Am J Clin Dermatol. 2004;5(3):199-203.

19. Jeong K-H, Oh S-J, Chon S, Lee M-H. Generalized acanthosis nigricans related to type $\mathrm{B}$ insulin resistance syndrome: a case report. Cutis. 2010;86(6):299-302.

20. Katz RA. Treatment of acanthosis nigricans with oral isotretinoin. Arch Dermatol. 1980;116(1):110-111. http://www.ncbi.nlm.nih.gov/ pubmed/7352756. Accessed October 27, 2017.

21. Walling HW, Messingham M, Myers LM, Mason CL, Strauss JS. Improvement of acanthosis nigricans on isotretinoin and metformin. J Drugs Dermatol. 2003;2(6):677-681.

22. Sriboonnark L, Arora H, Falto-Aizpurua L, Choudhary S, Connelly EA. Costello syndrome with severe nodulocystic acne: unexpected significant improvement of acanthosis nigricans after oral isotretinoin treatment. Case Rep Pediatr. 2015;2015:1-4.

23. Özdemi 'r M, Toy H, Mevli toğlu İ, Demi rkesen C. Generalized idiopathic acanthosis nigricans treated with acitretin. Journal of Dermatological Treatment. 2009;17(1):54-56.

24. Pilkington T, Brogden RN. Acitretin. Drugs. 1992;43(4):597-627.
25. Tankova T, Koev D, Dakovska L, Kirilov G. Therapeutic approach in insulin resistance with acanthosis nigricans. Int J Clin Pract. 2002;56(8):578-581.

26. Atabek ME, Pirgon O. Use of metformin in obese adolescents with hyperinsulinemia: a 6-month, randomized, double-blind, placebo-controlled clinical trial. J Pediatr Endocrinol Metab. 2008;21(4):339-348.

27. Lee P, Cranston I, Amiel S, Rahilly S, Green A. Effect of metformin on glucose disposal and hyperinsulinemia in a 14-year-old boy with acanthosis nigricans. Horm Res. 1997;48:88-92.

28. Bellot-Rojas P, Posadas-Sanchez R, Caracas-Portilla N, et al. Comparison of metformin versus rosiglitazone in patients with acanthosis nigricans: a pilot study. J Drugs Dermatol. 2006;5(9):884-889.

29. Barbato MT, Criado PR, Silva AK, Averbeck E, Guerine MB, Sá NB. Association of acanthosis nigricans and skin tags with insulin resistance. An Bras Dermatol. 87(1):97-104.

30. Roemmich JN, Clark PA, Lusk M, et al. Pubertal alterations in growth and body composition. VI. Pubertal insulin resistance: relation to adiposity, body fat distribution and hormone release. Int JObes. 2002;26(5):701-709.

31. Sinha S, Schwartz RA. Juvenile acanthosis nigricans. J Am Acad Dermatol. 2007;57(3):502-508.

32. Adderley-Rolle EM, Peter S. Regression of acanthosis nigricans with the addition of sitagliptin and pioglitazone. West Indian Med J. 2015;64(2):160-161.

33. Lunetta M, Di Mauro M, Le Moli R, Burrafato S. Long-term octreotide treatment reduced hyperinsulinemia, excess body weight and skin lesions in severe obesity with acanthosis nigricans. J Endocrinol Invest. 1996;19(10):699-703.

34. Sherertz EF. Improved acanthosis nigricans with lipodystrophic diabetes during dietary fish oil supplementation. Arch Dermatol. 1988;124(7):1094-1096.

35. Epstein E. Podophyllin therapy in acanthosis nigricans. J Invest Dermatol. 1951;17(1):7.

36. Rosenbach A, Ram R. Treatment of acanthosis nigricans of the axillae using a long-pulsed (5-msec) alexandrite laser. Dermatologic Surg. 2004;30(8):1158-1160.

37. Pirgon Ö, Sandal G, Gökçen C, Bilgin H, Dündar B. Social anxiety, depression and self-esteem in obese adolescent girls with acanthosis nigricans. J Clin Res Pediatr Endocrinol. 2015;7(1):63-68.

38. Coates P, Shuttleworth D, Rees A. Resolution of nicotinic acid-induced acanthosis nigricans by substitution of an analogue (acipimox) in a patient with type V hyperlipidaemia. Br J Dermatol. 1992;126(4):412-414.
Clinical, Cosmetic and Investigational Dermatology

\section{Publish your work in this journal}

Clinical, Cosmetic and Investigational Dermatology is an international, peer-reviewed, open access, online journal that focuses on the latest clinical and experimental research in all aspects of skin disease and cosmetic interventions. This journal is included on PubMed. The manuscript management system is completely online

\section{Dovepress}

and includes a very quick and fair peer-review system, which is all easy to use. Visit http://www.dovepress.com/testimonials.php to read real quotes from published authors 